\title{
ANT2 suppression by shRNA may be able to exert anticancer effects in HCC further by restoring SOCS1 expression
}

\author{
JI-YOUNG JANG ${ }^{1}$, YOON-KYUNG JEON ${ }^{1,2}$, CHOONG-EUN LEE ${ }^{3}$ and CHUL-WOO KIM ${ }^{1,2}$ \\ ${ }^{1}$ Tumor Immunity Medical Research Center, Cancer Research Institute, Seoul National University \\ College of Medicine, Seoul 110-799; ${ }^{2}$ Department of Pathology, Seoul National University \\ College of Medicine, Seoul 110-799; ${ }^{3}$ Laboratory of Immunology, Department of \\ Biological Science, Sungkyunkwan University, Suwon 440-746, Republic of Korea
}

Received July 12, 2012; Accepted September 26, 2012

DOI: $10.3892 /$ ijo.2012.1736

\begin{abstract}
Suppressor of cytokine signaling 1 (SOCS1) is a negative regulator of Janus kinase and the signal transducer and activation of transcription (Jak-STAT) pathway. SOCS-1 is known to be silenced by aberrant promoter methylation in human hepatocellular carcinoma (HCC) during early tumorigenesis, therefore, a strategy to restore SOCS1 expression can be utilized for cancer therapy. Here, we examined the influence of adenine nucleotide translocase 2 (ANT2) suppression by shorthairpin RNA (shRNA) on SOCS1 expression and its downstream effect in HCC. ANT2 shRNA treatment led to restoration of SOCS1 expression along with its promoter demethylation in Hep3B cells, which was accompanied by decreased DNA methyltransferase 1 (DNMT1) activity through the suppression of Ras/PI3K/Akt signaling. Restoration of SOCS1 by ANT2 knockdown, subsequently, inhibited STAT3 activity and downregulated the expression of miR-21, which has been reported to be an important onco-miR in HCC. Downregulation of miR-21 efficiently suppressed Hep3B cell proliferation in vitro with a comparable level to ANT2 shRNA treatment. ANT2 suppression by shRNA may be able to exert anticancer effects in HCC further by restoring SOCS1 expression.
\end{abstract}

Correspondence to: Professor Chul-Woo Kim, Department of Pathology, Seoul National University College of Medicine, 28 Yongon-dong, Jongno-gu, Seoul 110-799, Republic of Korea E-mail: cwkim@snu.ac.kr

Professor Choong-Eun Lee, Department of Biological Science, Sungkyunkwan University, Suwon 440-746, Republic of Korea E-mail: celee@skku.edu

Abbreviations: ANT2, adenine nucleotide translocase-2; RNAi, RNA interference; shRNA, short-hairpin RNA; siRNA, small-interfering RNA; SOCS1, suppressor of cytokine signaling-1; Jak-STAT, janus kinase and signal transducer and activation of transcription; HCC, human hepatocellular carcinoma

Key words: adenine nucleotide translocator-2, RNA interference, short-hairpin RNA, small-interfering RNA, suppressor of cytokine signaling-1, janus kinase and signal transducer and activation of transcription, human hepatocellular carcinoma

\section{Introduction}

Hepatocellular carcinoma (HCC) is one of the most prevalent and lethal types of cancer in Asia and its incidence is increasing. In Southeastern Asia, the majority of HCCs develops in individuals with chronic HBV or HCV infection (1). Chronic liver disease and liver cirrhosis is considered to be a premalignant state because HCC occurs frequently in the background of liver cirrhosis (2). Currently the treatment modalities in advance HCC is very limited, and therefore development of new strategy for HCC therapy based on molecular pathogenesis is necessary (3).

Adenine nucleotide translocase (ANT), abundantly located in the inner mitochondrial membrane, participates in the formation of mitochondrial permeability transition pore complex, and also plays an important role in the cellular energy metabolism by catalyzing the exchange of mitochondrial ATP for cytosolic ADP and thereby influencing mitochondrial oxidative phosphorylation $(4,5)$. Human ANT has four isoforms (ANT1, ANT2, ANT3 and ANT4) and the relative expressions of these isoforms are dependent on developmental stages, proliferation status as well as tissue types or cell types. ANT2 is specifically expressed in undifferentiated cells or tissues that are able to proliferate and regenerate; for example, the lymphocytes, kidney and liver $(6,7)$. The expression of ANT2 was recently found to be upregulated in several hormone-dependent cancers (8) and the induction of ANT2 expression in cancer cells was directly associated with glycolytic metabolisms, raising a question regarding the role of ANT2 during carcinogenesis (9-12). ANT2 suppression by a DNA vector-based RNA interference approach expressing short hairpin RNA (shRNA) resulted in ATP depletion and induced cell death in breast cancer cells (13). We also confirmed that the knockdown of ANT2 by shRNA induced cell death in HCC cell lines and screened for the identification of ANT2 shRNA regulated genes in the hepatocellular carcinoma cell line Hep3B. As a result, we obtained significant candidate genes and reaffirmed by selecting a specific gene, suppressor of cytokine signaling 1 (SOCS1), among the genes.

Involvement of SOCS1 in carcinogenesis revealed that SOCS1 is frequently silenced by hypermethylation in HCC (14). Restoration of SOCS1 in HCC cell lines by tran- 
sient transfection resulted in growth suppression and induction of apoptosis (14). The silencing of gene transcription associated with aberrant DNA methylation of CpG dinucleotides in normally unmethylated gene promoter regions is the most widely studied epigenetic abnormality in tumorigenesis (15-17). SOCS1 is a negative regulator of Janus kinase and signal transducer and activation of transcription (Jak-STAT) pathway (18-21). Several studies have indicated that dysregulation of the Jak-STAT pathway is involved in the malignant transformation for commonly-encountered human cancers, such as HCC (14) non-small cell lung cancer, and head and neck squamous cell carcinoma (22-24). The high prevalence of the aberrant SOCS1 methylation demonstrated the importance of the constitutive activation of the Jak-STAT pathway in the development of HCC (25).

Constitutive activated STAT3 controls the expression of a number of anti-apoptotic proteins on the transcriptional level, including survivin, Bcl-2 and Mcl-1, thereby providing an important fast-track survival signal for tumor cells (26-29). According to recent report, miR-21 was also identified as a target of STAT3. siRNA against STAT3 has much reduced levels of miR-21 (30). Moreover, STAT3-induced miR-21 was important for transformation and tumorigenicity in human cancer and inhibition of miR-21 dramatically reduced tumorigenicity (30). In HCC tissues, miR-21 was markedly increased compared with normal tissues and increased miR-21 expression was a frequent event in human HCC and may be involved in hepatocarcinogenesis. More importantly, the relevance of miR-21 for the oncogenic potential of STAT3 and thereby for its involvement in the pathogenesis of malignancies. Our results show that ANT2 shRNA treatment led to restoration of SOCS1 expression along with its promoter de-methylation in Hep3B cells.

We hypothesized that suppression of Ras/PI3K/Akt signaling by ANT2 shRNA induced SOCS1 restoration. It is well known that Ras/PI3K/Akt pathway elevates the cellular DNMT1 protein level. Upregulation of DNMT1 by Ras/PI3K/Akt signal pathway involves transcription activation and mRNA stabilization, and that DNMT1 protein stability was significantly decreased by Ras/PI3K/Akt inhibition. Eventually, epigenetic silencing was decreased through the suppression of Ras/PI3K/Akt signaling by ANT2 shRNA. Therefore, inhibition of DNMT1 activity by suppression of Ras/PI3K/Akt pathway decreases hyperme-thylated SOCS1 levels.

We assumed that SOCS1 restoration by ANT2 shRNA might inactivate Jak-STAT signaling pathway, thereby downregulating onco-miR-21 expression and subsequent inhibition of proliferation, in HCC cells. Thus, the purpose of our study was to determine whether or not ANT2 shRNA-mediated SOCS1 restoration suppressed HCC cells proliferation, and to investigate the potential of ANT2 shRNA for future use as a therapeutic agent targeting HCC cells.

\section{Materials and methods}

Cell lines and culture. The human hepatoma cell line Hep3B was purchased from the American Type Culture Collection (ATCC, Manassas, VA, USA) and cultured in Dulbecco's modified Eagle's medium supplemented with $10 \%$ fetal bovine serum, $100 \mathrm{U} / \mathrm{ml}$ penicillin and $100 \mu \mathrm{g} / \mathrm{ml}$ streptomycin (Gibco, Grand
Island, $\mathrm{NY}, \mathrm{USA}$ ) in a humidified $5 \% \mathrm{CO}_{2} / 95 \%$ air atmosphere at $37^{\circ} \mathrm{C}$.

Antibodies and reagents. Anti-phospho-STAT3, anti-STAT3, anti-SOCS1, anti-phospho-Akt, anti-Akt and anti- $\beta$-actin antibodies were purchased from Cell Signaling Technology (Beverly, MA, USA).

Construction of ANT2 shRNA expression vectors. The ANT2 shRNA expression vector used to achieve specific downregulation of ANT2 was described previously. ANT2 siRNA (5'-GCAG AUCACUGCAGAUAAGTT-3') designed to be complementary to exon 2 of ANT2 (GenBank accession no. NM001152) was synthesized, and DNA vectors expressing the shRNA forms of the siRNAs were generated using pSilencer 3.1-H1 puro plasmids with a TTCAAGAGA linker sequence that forms looped structures (Ambion, Austin, TX, USA). The vector expressing ANT2 shRNA was used throughout this study. A scramble shRNA (Ambion) with no significant homology to human gene sequences was used as a control to detect nonspecific effects.

Transfection. For transfection, cells were plated on either 6-well plates $\left(2 \times 10^{5}\right.$ cells/well $)$ or $100-\mathrm{mm}$ dishes $\left(2 \times 10^{6}\right.$ cells $)$ and were allowed to adhere for $24 \mathrm{~h}$. Lipofectamine 2000 (Invitrogen, Carlsbad, CA, USA) was used for the transfection. Cells were transfected with either pSilencer ${ }^{\mathrm{TM}}$ 3.1-H1 puro ANT2 shRNA or pSilencer $^{\mathrm{TM}}$ 3.1-H1 puro scramble shRNA vectors. Transfected cells were then cultured for $6 \mathrm{~h}$; culture media were replaced with fresh media supplemented with $10 \%$ FBS. The cells were harvested 24 or $48 \mathrm{~h}$ after transfection. pcDNA, pcDNA-ANT2, daRas, dnRas plasmid or SOCS1 shRNA were transfected into the cells using the same method.

Microarray analysis and statistical analysis. Isolated RNA (500-1,000 ng) was converted to cDNA with reverse transcriptase and an oligo (dT) primer bearing a T7 promoter followed by in vitro transcription with T7 RNA polymerase to create amplified antisense RNA. The amplified RNA was labeled with $\mathrm{Cy} 3$ or $\mathrm{Cy} 5$. As a reference probe universal human reference RNA (UHR, Stratagene) was amplified and labeled as above. Amplification and labeling efficiency was controlled using NanoDrop. Labeled cRNA was hybridized to Agilent Whole Human Genome Oligo Microarrays per the manufacturer's protocol. After hybridization for $17 \mathrm{~h}$ the arrays were washed and scanned using an Agilent scanner and microarray data extracted with Agilent Feature Extraction software.

Quantitative real-time RT-PCR (RT-qPCR). For the RT-qPCR, total-RNA from the same samples used in microarray analysis was tested by using Bio-Rad iCycler IQ real-time PCR system. PCR primers were designed with Primer Express 2.0 software (Invitrogen). Results are shown as fold change. RT-qPCR was carried out with the PrimerScript RT reagent Kit (Takara, Otsu, Japan) and SYBR-Green real-time PCR Master mix (Takara) according to manufacturer's instruction. The housekeeping gene GAPDH was used for standardization of the initial RNA content of a sample. Relative changes of gene expression were calculated by the following formula, and the data are represented as fold 
upregulation/downregulation, fold change $=2^{-\Delta \Delta \mathrm{Ct}}$, where $\Delta \Delta \mathrm{Ct}$ $=(\mathrm{Ct}$ of gene of interest, treated $-\mathrm{Ct}$ of $\mathrm{HK}$ gene, treated $)-(\mathrm{Ct}$ of gene of interest, control-Ct of HK gene, control), $\mathrm{Ct}$ was the threshold cycle number and HK was the house-keeping gene.

Western blot analysis. For western blot analyses, cells were harvested after 24 or $48 \mathrm{~h}$ of transfection and lysed with lysis buffer $(5 \mathrm{mM} / 1$ ethylenediamine tetra acetic acid; $300 \mathrm{mM} / 1$ $\mathrm{NaCl} ; 0.1 \%$ NP-40; $0.5 \mathrm{mM} / 1 \mathrm{NaF} ; 0.5 \mathrm{mM} / 1 \mathrm{Na}_{3} \mathrm{VO}_{4} ; 0.5 \mathrm{mM} / \mathrm{l}$ phenylmethylsulfonyl fluoride; and $10 \mu \mathrm{g} / \mathrm{ml}$ each of aprotinin, pepstatin and leupeptin; Sigma, St. Louis, MO, USA). Following centrifugation at $15,000 \times \mathrm{g}$ for $30 \mathrm{~min}$, the concentrations of supernatant proteins were analyzed using the Bradford reagent (Bio-Rad, Hercules, CA, USA). For analysis of protein contents, $50 \mu \mathrm{g}$ of total proteins was electrophoresed in $10 \%$ SDS-PAGE gel and transferred to polyvinylidene difluoride membranes (Millipore, Bedford, MA, USA), which were then incubated with the respective antibodies indicated above. Immunoblots were visualized using an enhanced chemiluminescence detection system (Amersham Pharmacia Biotech, Uppsala, Sweden).

Methylation-specific PCR (MSP). Genomic DNA was obtained and purified using QIAamp DNA mini Kits (Qiagen, Hilden, Germany). The unmethylated cytosines in aliquots containing 500 ng DNA were converted to uracil using MethylCode Bisulfite Conversion Kits (Invitrogen). We modified the manufacturer's instructions by extending the desulfonation step to $30 \mathrm{~min}$ to ensure complete conversion of the genomic DNA and by prewarming the elution buffer to $50^{\circ} \mathrm{C}$ to increase the DNA yield. Methylation-specific PCR for SOCS1 was performed using the primer pair 5'-TTC GCG TGT ATT TTT AGG TCG GTC-3' (forward) and 5'-CGA CAC AAC TCC TAC AAC GAC CG-3' (reverse) for methylated DNA and the pair 5'-TTA TGA GTA TTT GTG TGT ATT TTT AGG TTG GTT-3' (forward) and 5'-CGA CAC AAC TCC TAC AAC GAC CG-3' (reverse) for unmethylated DNA. PCR mixtures contained 1X PCR buffer, $2 \mathrm{mM} \mathrm{MgCl}_{2}, 200 \mathrm{mM} \mathrm{dNTPs}, 200 \mathrm{nM}$ of each primer, $0.05 \mathrm{U} / \mathrm{ml}$ Taq polymerase and $6 \mathrm{ng} / \mathrm{ml}$ converted DNA as the template. PCR products were resolved in $2 \%$ agarose gels in $1 \mathrm{X}$ Tris/borate/EDTA buffer and stained with ethidium bromide.

Reverse transcription-polymerase chain reaction (RT-PCR). Cells were collected after $24 \mathrm{~h}$ of transfection. Total-RNA was extracted using TRIzol (Invitrogen), according to the manufacturer's instructions. For RT-PCR analysis, $5 \mu \mathrm{g}$ total-RNA was reverse-transcribed using RT-PCR kits (Promega, Madison, WI, USA). PCR was used for amplification of target cDNA under the following conditions: 35 cycles of $94^{\circ} \mathrm{C}$ for $1 \mathrm{~min}, 55^{\circ} \mathrm{C}$ for $1 \mathrm{~min}$ and $72^{\circ} \mathrm{C}$ for $2 \mathrm{~min}$. PCR products were analyzed using standard agarose gel electrophoresis. The primer sequences were: for SOCS1, forward 5'-TTGCCTGGAACCATGTGG-3' and reverse 5'-GGTCCTGGCCTCCAGATACAG-3'; for $\beta$-actin, forward 5'-GCTCCG GCATGTGCAA-3' and reverse 5'-GCTC CGGCATGTGCAA-3'.

DNA methyltranserase 1 (DNMT1) activity assay. Nuclear extracts from Hep3B cells transfected for $24 \mathrm{~h}$ with ANT2 or scramble shRNA (control) were evaluated for DNMT1 activity using an EpiQuik Dnmt1 Assay Kit (Epigentek Inc., Brooklyn, NY, USA).
Electrophoretic mobility shift assay (EMSA). Nuclear extracts of Hep3B transfected with scramble or ANT2 shRNA, were prepared and EMSA performed. In brief, for EMSA, $3.4 \mu \mathrm{g}$ of total nuclear protein were used for each lane. The oligonucleotide sequence used to assess the STAT3 consensus-binding motif 5'-GATCCTTCTGGGAATTCCTAGATC-3'. All oligos were synthetized by Bioneer (Dajeon, Korea). The synthetic oligonucleotides were labeled using a T4 polynucleotide kinase (DNA 5'-End Labeling Kit; Promega, Mannheim, Germany) and $\left[\gamma^{32} \mathrm{P}\right]$-CTP (Perkin Elmer, Rodgau-Jügesheim, Germany). Following incubation of the radiolabeled probes with nuclear extracts, protein-DNA complexes were resolved on a NOVEX $6 \%$ retardation gel (Invitrogen) and detected using a Typhoon 9410 PhosphoImager (Amersham Biosciences, Freiburg, Germany).

Chromatin immunoprecipitation (ChIP) assays. ChIP assay was carried out using the ChIP-IT Express Enzymatic kit (Active Motif) according to the manufacturer's instructions. In brief, chromatin from cells was cross-linked with $1 \%$ formaldehyde $\left(10 \mathrm{~min}\right.$ at $\left.22^{\circ} \mathrm{C}\right)$, sheared to an average size of $\sim 500 \mathrm{bp}$ and then immunoprecipitated with anti-STAT3 antibody (Santa Cruz Biotechnology). The ChIP-PCR primers were designed to amplify a proximal promoter region containing putative binding sites in the miR-21 promoter identified by TFSEARCH.

MTT cell proliferation assay. Cell proliferation was measured using the MTT assay (Sigma). The reduction of tetrazolium salts is now widely accepted as a reliable way to examine cell proliferation. The yellow tetrazolium MTT (3-(4, 5-dimethylthiazolyl-2)-2, 5-diphenyltetrazolium bromide) is reduced by metabolically active cells, in part by the action of dehydrogenase enzymes, to generate reducing equivalents such as NADH and NADPH. The resulting intracellular purple formazan can be solubilized and quantified by spectrophotometric means. Results of cell proliferation assays are presented as the mean values of three replicate experiments performed in triplicate.

\section{Results}

ANT2 shRNA induces specific restoration of SOCS1 through the demethylation of SOCS1 promoter in the hepatocellular carcinoma cell line Hep3B. We first microarray screened for the identification of ANT2 shRNA regulated genes in the hepatocellular carcinoma cell line Hep3B (Fig. 1A). We obtained significant candidate genes and reaffirmed by selecting a specific gene, SOCS1, among the genes. The SOCS1 gene has been demonstrated to be frequently silenced by methylation of the $\mathrm{CpG}$ islands in human HCC. As shown in Fig. 1B, SOCS1 was upregulated by ANT2 shRNA. However, ANT2 shRNA had little influence on SOCS3 expression. These data suggested that ANT2 shRNA upregulated the SOCS1 expression specifically in Hep3B. Furthermore, we observed that ANT2 shRNA decreased hyper-methylated levels and increased unmethylated levels of SOCS1 and that dominant active Ras suppressed unmethylated levels of SOCS1 and recovered reduction of hyper-methylated levels of SOCS1 in Hep3B cells (Fig. 2A). In SOCS1 expression, the result matched those in Fig. 2B. Thus, SOCS1 upregulation in response to ANT2 knockdown is mediated by SOCS1 promoter demethylation. 
A

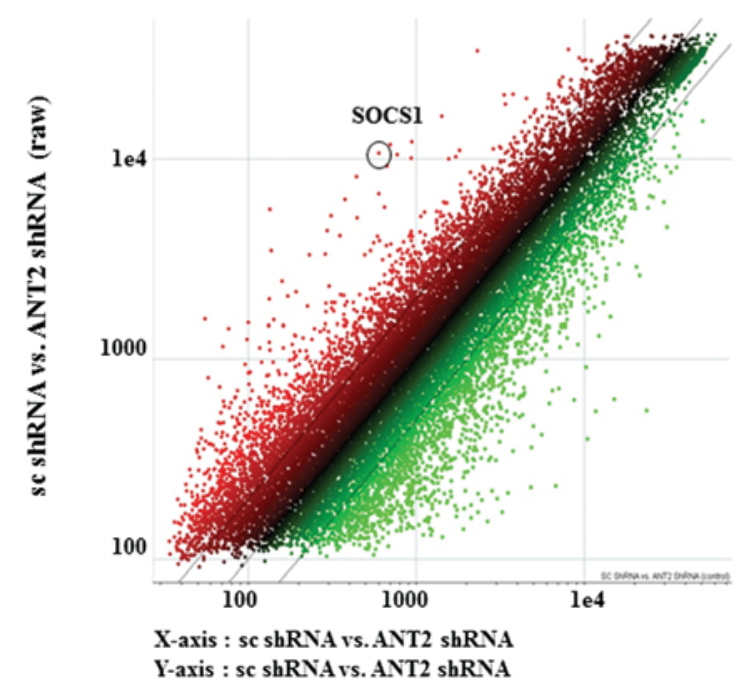

B

[Real time-PCR]

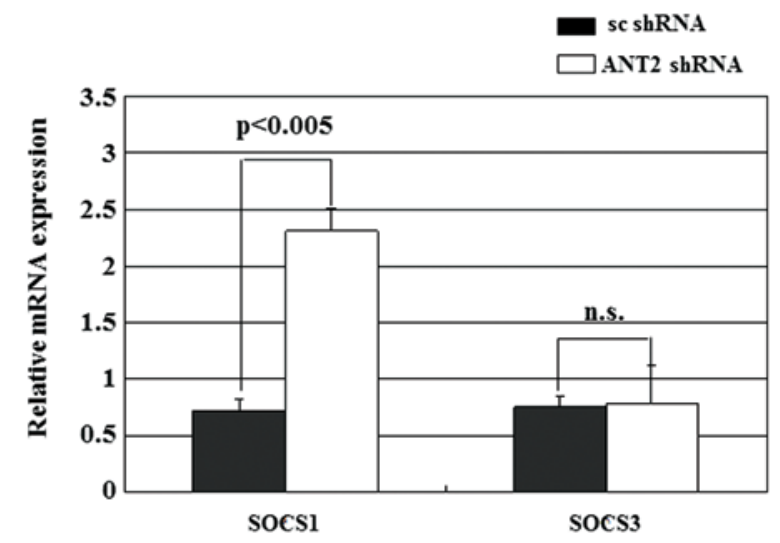

Figure 1. Knockdown of ANT2 by shRNA induces specific restoration of SOCS1 in Hep3B. (A) RNA extraction was performed $24 \mathrm{~h}$ after transfection of Hep3B cells with ANT2 shRNA for microarray analysis. Isolated RNA was converted to cDNA and amplified. The amplified RNA was labeled with Cy3 or Cy5. Labeled cRNA was hybridized to Agilent Whole Human Genome Oligo Microarrays per the manufacturer's protocol. After hybridization for $17 \mathrm{~h}$ the arrays were washed and scanned using an Agilent scanner and microarray data extracted with Agilent Feature Extraction software. (B) RT-qPCR analysis of the effect of ANT2 shRNA-induced restoration on SOCS1 expression. Cells were transfected with scramble or ANT2 shRNA. Total-RNA was extracted $24 \mathrm{~h}$ after transfection and subjected to RT-qPCR using specific primers for human SOCS1, SOCS3 or glyceraldehyde phosphate dehydrogenase (GAPDH, internal control).

A

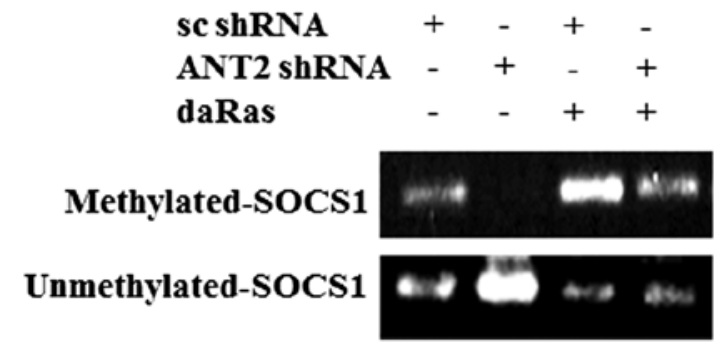

[Methylation Specific PCR]
B

[RT-PCR]

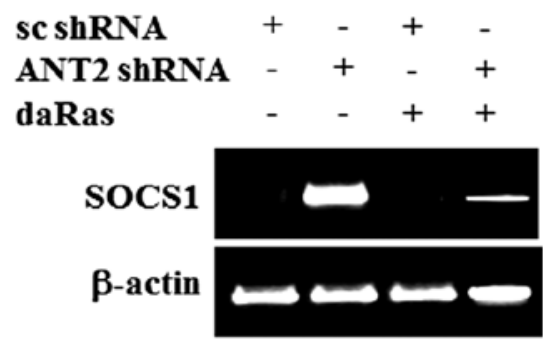

Figure 2. ANT2 shRNA induces restoration of SOCS1 through the demethylation of SOCS1 promoter. (A) Methylation-specific PCR analysis of effect of ANT2 shRNA on SOCS1 genomic DNA of Hep3B cells. After cells were transfected with scrambled, ANT2 shRNA and/or dominant active Ras for 24 h, they were lysed and the genomic DNA was purified. Unmethylated cytosines were converted to uracils using an Invitrogen MethylCode Bisulfite Conversion kit and the converted genomic DNA was used for PCR with methylation-specific primers. The PCR products were subjected to electrophoresis on agarose gels and stained with ethidium bromide. (B) RT-PCR analysis of the effect of ANT2 shRNA-induced restoration on SOCS1 expression. Cells were transfected with scramble, ANT2 shRNA and/or dominant active Ras. Total-RNA was extracted $24 \mathrm{~h}$ after transfection and subjected to RT-PCR using specific primers for human SOCS1 or $\beta$-actin (internal control).

ANT2 shRNA reduces activity of DNA methyl-transferase 1 (DNMT1), by inhibiting Ras/PI3K/Akt signaling pathway in the hepatocellular carcinoma cell line Hep3B. Here, we examined whether ANT2 knockdown by shRNA affected activity of DNMT1 and whether this might be regulated by the Ras/PI3K/Akt signaling pathway. Ras protein is the most wellcharacterized proto-oncogene and Ras signaling is known to regulate diverse cellular functions including cell growth, survival and migration. Indeed, the level of DNA methyltransferase and DNA methylation are controlled by the RAS signaling pathway. Akt (also named protein kinase B) is a downstream target of Ras signaling pathway and is a critical mediator of survival signals for protection of cells from apoptosis. Especially, relationship between Ras/PI3K/Akt signaling and epigenetic silencing have been reported. Activated Ras/PI3K/Akt signaling is a trigger of epigenetic silencing. To detect the regulation of Ras/PI3K/ Akt activity by ANT2 shRNA, we examined phosphorylated Akt levels was decreased by knockdown of ANT2 by shRNA (Fig. 3A). A report that DNMT1 activation is involved in Ras/ PI3K/Akt signaling and our observations that ANT2 knockdown inactivates Ras/PI3K/Akt signaling and causes demethylation of the SOCS1 promoter in Hep3B cells, led us to investigate the role of DNMT1 in ANT2 shRNA-induced restoration of SOCS1. Besides, DNMT1 activity assays showed transfection with dominant active Ras restored the reduction of DNMT1 activity in Hep3B cells transfected with ANT2 shRNA and dominant 
$\mathbf{A}$

[Western blot]

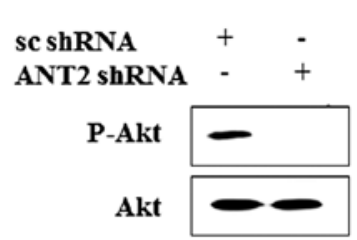

B

[DNMT1 activity assay]

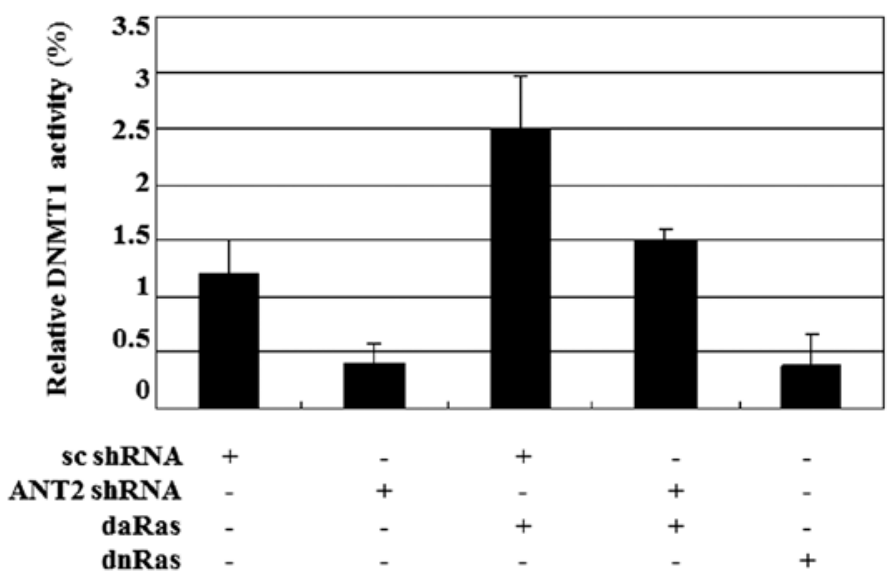

Figure 3. Demethylation of SOCS1 promoter by ANT2 shRNA is dependent on inactivation of DNMT1 through the suppression of Ras/PI3K/Akt signaling. (A) After $24 \mathrm{~h}$ of transfection with scramble shRNA or ANT2 shRNA, cells extracts were prepared for western blot analysis with anti-phospho-Akt and anti-Akt antibodies. (B) Effect of ANT2 shRNA-induced Ras signaling on DNMT1 activity. Hep3B cells were transfected with scrambled, ANT2 shRNA and/or dominant active Ras and nuclear extracts were evaluated for DNMT1 activity. Three independent experiments were performed and the data were analyzed using the Student's t-test.

$\mathbf{A}$

[Western blot]

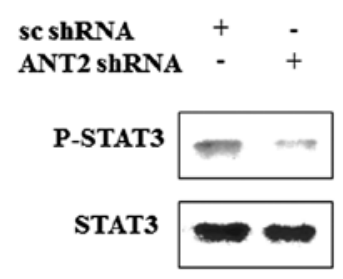

[EMSA]

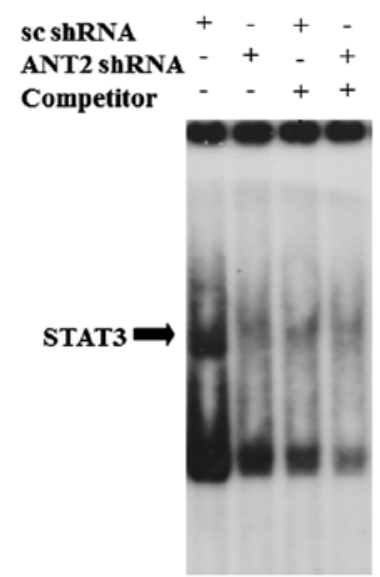

$\mathbf{B}$

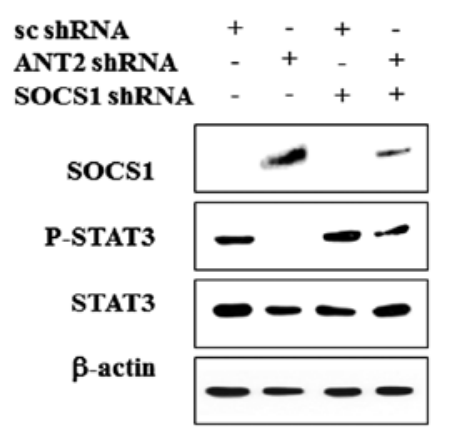

Figure 4. Restoration of SOCS1 by ANT2 shRNA inhibits phosphorylation and DNA binding activity of STAT3. (A) After $24 \mathrm{~h}$ of transfection with scramble shRNA or ANT2 shRNA, the cellular extracts were subjected to western blot analysis with anti-phospho-STAT3 and anti-STAT3 antibodies. Moreover, electrophoretic mobility shift assay (EMSA) was achieved by using DNA oligomer with consensus STAT3 binding sites. (B) After $24 \mathrm{~h}$ of transfection with scramble shRNA, ANT2 shRNA and/or SOCS1 shRNA, the cellular extracts were subjected to western blot analysis with SOCS1, anti-phospho-STAT3, anti-STAT3 and $\beta$-actin antibodies.

negative Ras also suppressed DNMT1 activity (Fig. 3B). Taken together, these findings suggested that ANT2 shRNA treatment led to restoration of SOCS1 expression along with its promoter demethylation in Hep3B cells, which was accompanied by decreased DNMT1 activity through suppression of Ras/PI3K/ Akt signaling.

Restoration of SOCS1 by ANT2 shRNA inhibits STAT3 activity. We observed that ANT2 shRNA transfection recovered SOCS1 expression levels in Hep3B cells. Because SOCS1 is known to a negative regulator of Janus kinase and signal transducer and activation of transcription (Jak-STAT) pathway, we anticipated that STAT3 might be inactivated by restored SOCS1 by ANT2 knockdown. As expected, ANT2 shRNA treatment inhibits phosphorylation and DNA binding activity of STAT3 (Fig. 4A). Furthermore, the phosphorylation of STAT3 in ANT2 shRNAtransfected cells was inhibited by the SOCS1 shRNA (Fig. 4B). Taken together, these findings suggest that ANT2 knockdown reduces hypermethylation of SOCS1 promoter to restore SOCS1 expression, which then inactivates the Jak-STAT signaling pathway.

Inactivation of STAT3 by ANT2 shRNAdownregulates onco-miR-21 expression. Because silencing of SOCS1 in hepatocellular carcinoma is attributable to enhanced activation of STAT3, as a result, induced proliferation and tumorigenesis. A STAT3 target gene that was described previously is the gene encoding the primary transcript of miR-21. To assay the suppression of the 
A

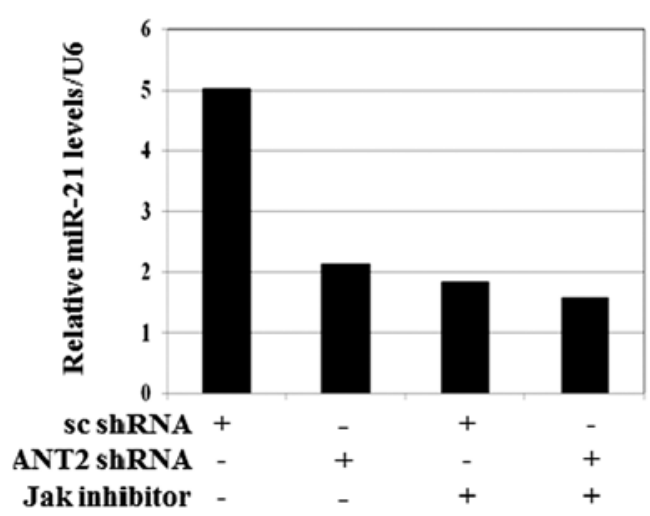

B [Chip assay]

MiR-21 \& STAT3 interaction

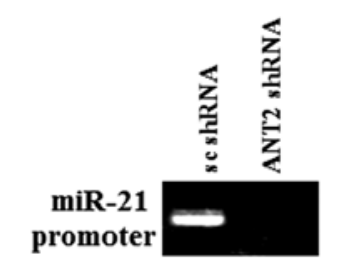

Input DNA (GAPDH)
C

[Western blot]
D

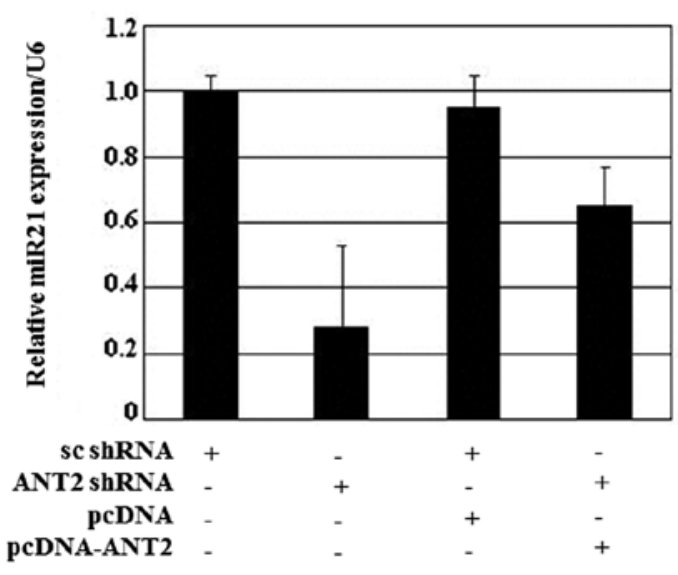

E

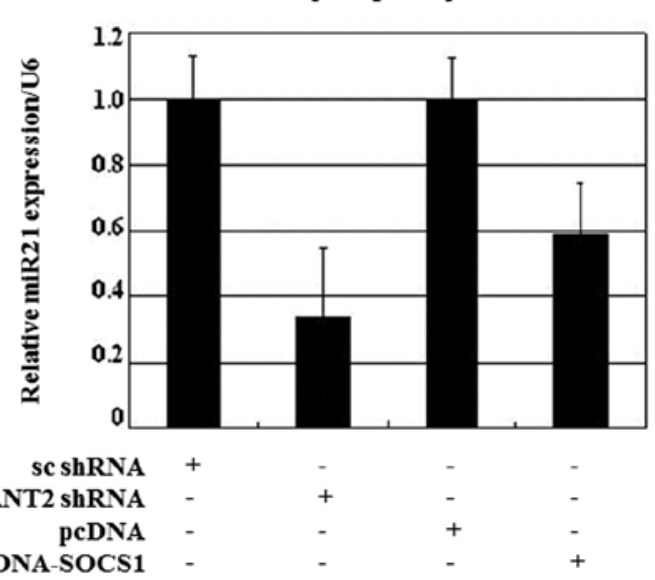

Figure 5. Restoration of SOCS1 by ANT2 shRNA, subsequently down-regulated the expression of onco-miR-21 through the inactivation of STAT3. (A) RT-qPCR analysis of the effect of ANT2 shRNA on the downregulation of miR-21 expression. Cells were transfected with scramble or ANT2 shRNA. Total-RNA was extracted $24 \mathrm{~h}$ after transfection and subjected to RT-qPCR using specific primers for human miR-21 or U6 (internal control). (B) After $24 \mathrm{~h}$ of transfection with scramble shRNA or ANT2 shRNA, the cellular extracts were subjected to chip assay. (C) After 24 h of transfection with scramble shRNA, ANT2 shRNA, pcDNA and/or pcDNA-ANT2, the cellular extracts were subjected to western blot analysis with anti-phospho-STAT3 and anti-STAT3 antibodies. (D) After $24 \mathrm{~h}$ of transfection with scramble shRNA, ANT2 shRNA, pcDNA and/or pcDNA-ANT2, total-RNA was extracted 24 h after transfection and subjected to RT-qPCR using specific primers for human miR-21 or U6 (internal control). (E) After $24 \mathrm{~h}$ of transfection with scramble shRNA, ANT2 shRNA, pcDNA and/or pcDNA-SOCS1, total-RNA was extracted $24 \mathrm{~h}$ after transfection and subjected to RT-qPCR using specific primers for human miR-21 or U6 (internal control).

miR-21 by ANT2 shRNA, quantitative reverse transcriptase PCR (RT-qPCR) analysis of RNA isolated from Hep3B cells transfected with scramble or ANT2 shRNA showed that the expression of miR-21 is suppressed by ANT2 shRNA (Fig. 5A). We investigated whether miR-21 is also a direct STAT3 target. Cells were transfected with ANT2 shRNA to inactivate STAT3, and analyzed by chromatin immunoprecipitation (ChIP). Chromatin that was precipitated with STAT3 antibodies was significantly enriched for the miR-21 promoter sequence when compared with chromatin precipitated with normal rabbit $\mathrm{IgG}$. As a control, PCR was performed on the precipitated chromatin for the promoter of glyceraldehyde3-phosphate dehydrogenase (GAPDH), a promoter that lacks STAT3 binding sites. No precipitation of the GAPDH promoter was observed with or without ANT2 shRNA, demonstrating the specificity of the ChIP (Fig. 5B). Collectively, restoration of SOCS1 by ANT2 knockdown, subsequently inhibited STAT3 activity and downregulated the expression of miR-21, which has been reported to be an important onco-miR in HCC and other carcinomas. Furthermore, this suppression of miR-21 by ANT2 shRNA was restored by ANT2 or SOCS1 overexpression (Fig. 5A and B) and reduction of phospho-STAT3 levels was restored by ANT2 overexpression (Fig. 5C). Moreover, reduction of miR-21 was recovered by ANT2 or SOCS1 overexpression in ANT2 shRNA transected Hep3B cells (Fig. 5D and E). These results suggested that inhibition of Jak-STAT signaling by ANT2 shRNA might play an important role in the downregulation of miR-21.

Downregulation of miR-21 by ANT2 shRNA inhibits cell proliferation. Reduction of miR-21 levels by miR-21 inhibitor inhibited proliferation. Similar inhibition of cell growth and proliferation effects was also observed in ANT2 shRNA transfected cells (Fig. 6A). Downregulation of miR-21 efficiently suppressed Hep3B cell proliferation in vitro with a comparable level to ANT2 shRNA treatment. Overall, the possible pathogenesis of miR-21 suppression by ANT2 shRNAinduced SOCS1 restoration is illustrated in Fig. 6B. 
$\mathbf{A}$

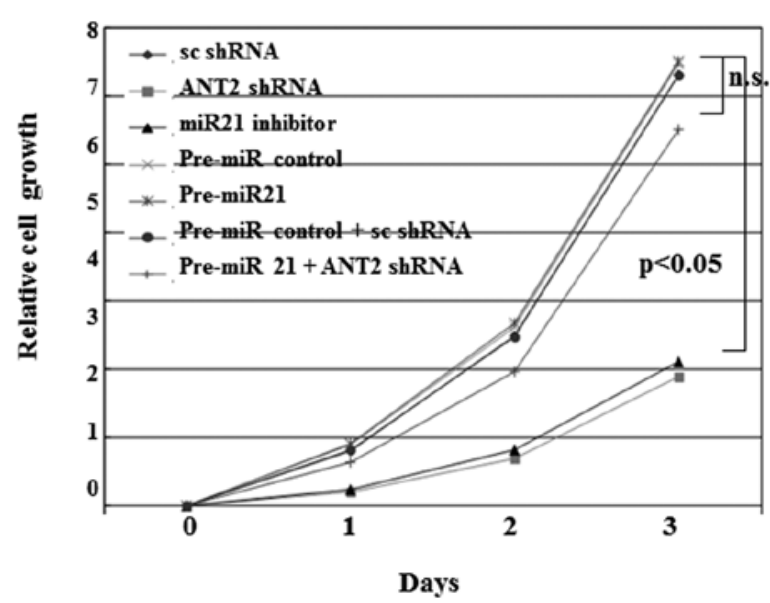

B

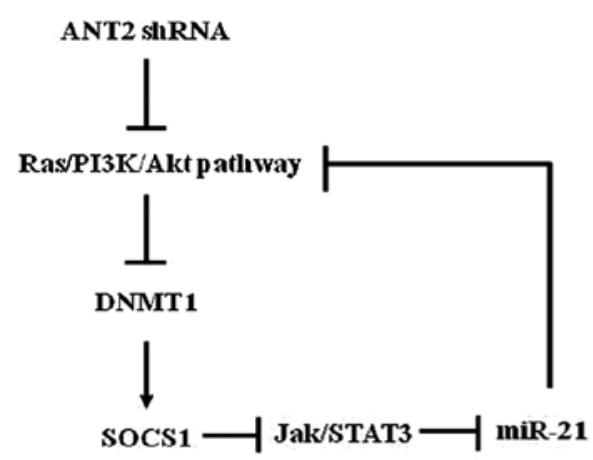

Figure 6. Downregulation of miR-21 by ANT2 shRNA inhibits cell proliferation. (A) Hep3B cells were transfected with scramble, ANT2 shRNA, pre-miR-control, pre-miR-21 or miR-21 inhibitor. Cell viability (MTT) assays were performed 24, 48 and $72 \mathrm{~h}$ after transfection. Three independent experiments were performed. Data were analyzed using the Student's t-test. (B) Schematic signaling pathways that are expected.

\section{Discussion}

Recently, the potential role of the Jak/STAT pathway in oncogenesis has been proposed in many kinds of tumors $(31,32)$. The SOCS family has been identified as a negative feedback protein of Jak/STAT signaling pathway. These proteins are activated by STATs and negatively regulate the Jak/STAT pathway by inhibiting the Jaks directly or blocking the access of the STATs (33). Among the SOCS family molecules, the SOCS1 expression was suppressed through aberrant methylation of the $\mathrm{CpG}$ islands in several HCC studies. Promoter methylation in the SOCS1 CpG islands was identified in primary HCC. Moreover, SOCS1 methylation was observed more frequently in HCCs derived from cirrhosis than in those not derived from cirrhosis. This report suggests that inactivation of SOCS1 might be an important factor in carcinogenesis of $\operatorname{HCC}(25,34)$.

The overexpression of ANT2 in cancer cells was found to be associated with glycolytic metabolism of cancer cells, which raised a possible role of ANT2 during carcinogenesis (10). In fact, we and others have shown that ANT2 knockdown decreased cell viability and induced apoptotic death in cancer cells and also can sensitize cancer cells to a kind of chemotherapeutic agents $(8,13)$.

In the work shown here, knockdown of ANT2 by shRNA restored SOCS1 expression and suppressed HCC cell proliferation. We also observed that ANT2 shRNA treatment led to restoration of SOCS1 expression along with its promoter demethylation in Hep3B cells, which was accompanied by decreased DNA methyl-transferase 1 (DNMT1) activity through suppression of Ras/PI3K/Akt signaling. Generally, DNMT1 is overexpressed in several cancers and upregulated by oncogenic pathways and downregulated by tumor suppressive pathways (35-37). Recently, prolonged half-life of DNMT1 protein in cancer has also been reported, the mechanism of its regulation is found that Akt activity positively correlates with DNMT1 protein level but not mRNA level. Akt is a serine/threonine protein kinase activated downstream of phosphatidylinositol 3-kinase (PI3K). The mechanism attributed to epigenetic role of
PI3K/Akt in regulating protein stability of a pivotal epigenetic molecule-DNMT1. Activated PI3K/Akt signaling greatly stabilizes DNA methyltransferase 1 (DNMT1) by attenuating the ubiquitin proteosome reaction in cancer cells (38). In this study, we observed that the suppression of Ras/PI3K/Akt signaling by ANT2 shRNA induced SOCS1 restoration through the inhibition of DNMT1.

Restoration of SOCS1 by ANT2 knockdown, subsequently, inhibited STAT3 activity and downregulated the expression of miR-21, which has been reported to be an important onco-miR in HCC. Downregulation of miR-21 efficiently suppressed Hep3B cell proliferation in vitro with a comparable level to ANT2 shRNA treatment. We demonstrated here for the first time that ANT2 shRNA induces suppression of proliferation, accompanied by inactivation of STAT3 through restoration of SOCS1 in HCC cell lines.

According to another report, the restoration of SOCS1 suppressed both growth rate and anchorage-independent growth of cells in which SOCS1 was methylation-silenced and Jak2 was constitutively activated (14). This growth suppression was caused by apoptosis and was reproduced by AG490, a specific, chemical Jak2 inhibitor that reversed constitutive phosphorylation of STAT3 in SOCS1 inactivated cells. The high prevalence of the aberrant SOCS1 methylation and its growth suppression activity demonstrated the importance of the constitutive activation of the Jak/STAT pathway in the development of HCC. It also indicates therapeutic strategies for the treatment of HCC including use of SOCS1 in gene therapy and inhibition of Jak2 by small molecules, such as AG490 (14). In the present study, ANT2 shRNA suppressed onco-miR-21 expression and STAT3 activition and preceded restoration of SOCS1. We thus hypothesized that ANT2 shRNA may also inhibit the Jak/STAT signaling pathway through the restoration of SOCS1, which may explain why ANT2 shRNA can suppress the growth rate of the HCC cell line. This study provides strong evidence that inactivation of Jak/STAT signaling pathway by ANT2 shRNA downregulates onco-miR-21 expression as well as inhibits proliferation. 
ANT2 suppression by shRNA might be able to exert anticancer effect in HCC further by restoring the SOCS1 expression.

miRNAs play important roles in multiple biological processes such as development, differentiation, and cellular stress response (39). Recent studies have linked deregulation of miRNAs to various diseases including cancer (40). According to recent reports, dramatic upregulation of miR-21 is observed in HCC and dysregulation of miR-21 is associated with HCC formation and progression. Moreover, uncontrolled proliferation, lack of apoptosis, and invasiveness, all modulated by miR-21, were also found to be present in tumorigenesis (41). Several questions remain unanswered. For example, miR-21 targets multiple negative regulators of Ras signaling, but the molecular alteration caused by miR-21-mediated Ras regulation remains unidentified (42). miR-21 is a potential onco-miR that plays important roles in HCC formation and progression. Moreover, a regulatory loop between Ras/PI3K/Akt pathway and miR-21 is mediated by STAT3. In conclusion, the ANT2 suppression by shRNA might be able to exert anticancer effect in HCC further by restoring the SOCS1 expression.

\section{Acknowledgements}

This work was supported by the Global Core Research Center (GCRC) grant (no. 2012-0001190) from the National Research Foundation (NRF), Ministry of Education, Science and Technology (MEST), Republic of Korea. This work was supported by grant (no. SS100003) from the Seoul R\&BD Program.

\section{References}

1. Yang JD and Roberts LR: Hepatocellular carcinoma: a global view. Nat Rev Gastroenterol Hepatol 7: 448-458, 2010.

2. Llovet JM, Burroughs A and Bruix J: Hepatocellular carcinoma. Lancet 362: 1907-1917, 2003.

3. Blum HE: Hepatocellular carcinoma: therapy and prevention. World J Gastroenterol 11: 7391-7400, 2005.

4. Marzo I, Brenner C, Zamzami N, Susin SA, Beutner G, Brdiczka D, Remy R, Xie ZH, Reed JC and Kroemer G: The permeability transition pore complex: a target for apoptosis regulation by caspases and bcl-2-related proteins. J Exp Med 187: 1261-1271, 1998.

5. Marzo I, Brenner C, Zamzami N, Jurgensmeier JM, Susin SA, Vieira HL, Prevost MC, Xie Z, Matsuyama S, Reed JC and Kroemer G: Bax and adenine nucleotide translocator cooperate in the mitochondrial control of apoptosis. Science 281 : 2027-2031, 1998.

6. Dolce V, Scarcia P, Iacopetta D and Palmieri F: A fourth ADP/ATP carrier isoform in man: identification, bacterial expression, functional characterization and tissue. FEBS Lett 579: 633-637, 2005.

7. Lunardi $\mathrm{J}$ and Attardi G: Differential regulation of expression of the multiple ADP/ATP translocase genes in human cells. J Biol Chem 266: 16534-16540, 1991.

8. Le Bras M, Borgne-Sanchez A, Touat Z, El Dein OS, Deniaud A, Maillier E, Lecellier G, Rebouillat D, Lemaire C, Kroemer G, Jacotot $\mathrm{E}$ and Brenner C: Chemosensitization by knockdown of adenine nucleotide translocase-2. Cancer Res 66: 9143-9152, 2006.

9. Faure Vigny H, Heddi A, Giraud S, Chautard D and Stepien G: Expression of oxidative phosphorylation genens in renal tumors and tumoral cell lines. Mol Carcinog 16: 165-172, 1996.

10. Chevrollier A, Loiseau D, Chabi B, Renier G, Donay O, Malthiery Y and Stepien G: ANT2 isoform required for cancer cell glycolysis. J Bioenerg Biomembr 37: 307-316, 2005.

11. Chevrollier A, Loiseau D, Gautier F, Malthlery Y and Stepien G: ANT2 expression under hypoxic conditions produces opposite cell-cycle behavior in 143B and HepG2 cancer cells. Mol Carcinog 42: 1-8, 2005.
12. Chevrollier A, Loiseau D and Stepien G: What is the specific role of ANT2 in cancer cells? Med Sci (Paris) 21: 156-161, 2005 (In French).

13. Jang JY, Choi Y, Jeon YK and Kim CW: Suppression of adenine nucleotide translocase-2 by vector-based siRNA in human breast cancer cells induces apoptosis and inhibits tumor growth in vitro and in vivo. Breast Cancer Res 10: R11, 2008.

14. Yoshikawa H, Matsubara K, Qian GS, Jackson P, Groopman JD, Manning JE, Harris CC and Herman JG: SOCS-1, a negative regulator of the JAK/STAT pathway, is silenced by methylation in human hepatocellular carcinoma and shows growth-suppression activity. Nat Genet 28: 29-35, 2001.

15. Singal $\mathrm{R}$ and Ginder GD: DNA methylation. Blood 93: 4059-4070, 1999.

16. Robertson KD and Jones PA: DNA methylation: past, present and future directions. Carcinogenesis 21: 461-467, 2000.

17. Baylin SB, Esteller M, Rountree MR, Bachman KE, Schuebel K and Herman JG: Aberrant patterns of DNA methylation, chromatin formation and gene expression in cancer. Hum Mol Genet 10: 687-692, 2001.

18. Heinrich PC, Behrmann I, Muller-Newen G, Schaper F and Graeve L: Interleukin-6-type cytokine signaling through the gp130/Jak/STAT pathway. Biochem J 334: 297-314, 1998.

19. Imada $\mathrm{K}$ and Leonard WJ: The Jak-STAT pathway. Mol Immunol 37: 1-11, 2000.

20. Alexander WS, Starr R, Metcalf D, Nicholson SE, Farley A, Elefanty AG, Brysha M, Kile BT, Richardson R, Baca M, Zhang JG, Willson TA, Viney EM, Sprigg NS, Rakar S, Corbin J, Mifsud S, DiRago L, Cary D, Nicola NA and Hilton DJ: Suppressors of cytokine signaling (SOCS): negative regulators of signal transduction. J Leukoc Biol 66: 588-592, 1999.

21. Nicola NA and Greenhalgh CJ: The suppressors of cytokine signaling (SOCS) proteins: important feedback inhibitors of cytokine action. Exp Hematol 28: 1105-1112, 2000.

22. He B, You L, Uematsu K, Zang K, Xu Z, Lee AY, Costello JF, McCormick F and Jablons DM: SOCS-3 is frequently silenced by hypermethylation and suppresses cell growth in human lung cancer. Proc Natl Acad Sci USA 100: 14133-14138, 2003.

23. He B, You L, Xu Z, Mezieres J, Lee AY and Jablons DM: Activity of the suppressor of cytokine signaling-3 promoter in human non-small-cell lung cancer. Clin Lung Cancer 5: 366-370, 2004

24. Weber A, Hengge UR, Bardenheuer W, Tischoff I, Sommerer F, Markwarth A, Dietz A, Wittekind C and Tannapfel A: SOCS-3 is frequently methylated in head and neck squamous cell carcinoma and its precursor lesions and causes growth inhibition. Oncogene 24: 6699-6708, 2005.

25. Chu PY, Yeh CM, Hsu NC, Chang YS, Chang JG and Yeh KT: Epigenetic alteration of the SOCS1 gene in hepatocellular carcinoma. Swiss Med Wkly 140: w13065, 2010.

26. Isomoto $\mathrm{H}$, Kobayashi $\mathrm{S}$, Werneburg $\mathrm{NW}$, Bronk SF, Guicciardi ME, Frank DA and Gores GJ: Interleukin 6 upregulates myeloid cell leukemia-1 expression through a STAT3 pathway in cholangiocarcinoma cells. Hepatology 42: 1329-1338, 2005.

27. Konnikova L, Kotecki M, Kruger MM and Cochran BH: Knockdown of STAT3 expression by RNAi induces apoptosis in astrocytoma cells. BMC Cancer 3: 1-9, 2003.

28. Aoki Y, Feldman GM and Tosato G: Inhibition of STAT3 signaling induces apoptosis and decreases survivin expression in primary effusion lymphoma. Blood 101: 1535-1542, 2003.

29. Jourdan M, Veyrune JL, Vos JD, Redal N, Couderc G and Klein B: A major role for Mcl-1 antiapoptotic protein in the IL-6-induced survival of human myeloma cells. Oncogene 22: 2950-2959, 2003.

30. Iliopoulos D, Jaeger SA, Hirsch HA, Bulyk ML and Struhl K: STAT3 activation of miR-21 and miR-181b-1 via PTEN and CYLD are part of the epigenetic switch linking inflammation to cancer. Mol Cell 39: 493-506, 2010.

31. Lacronique V, Boureux A, Valle VD, Poirel H, Quang CT, Mauchauffe M, Berthou C, Lessard M, Berger R, Ghysdael J and Bernard OA: A TEL-JAK2 fusion protein with constitutive kinase activity in human leukemia. Science 278: 1309-1312, 1997.

32. Bromberg JF, Wrzeszczynska MH, Devgan G, Zhao Y, Pestell RG, Albanese C and Darnell JE: Stat 3 as an oncogene. Cell 98: 295-303, 1999.

33. Cooney RN: Suppressors of cytokine signaling (SOCS): inhibitors of the Jak/STAT pathway. Shock 17: 83-90, 2002. 
34. Okochi O, Hibi K, Sakai M, Inoue S, Takeda S, Kaneko T and Nakao A: Methylation-mediated silencing of SOCS-1 gene in hepatocellular carcinoma derived from cirrhosis. Clinical Cancer Res 9: 5295-5298, 2003.

35. Sun L, Hui AM, Kanai Y, Sakamoto M and Hirohashi S: Increased DNA methyltransferase expression is associated with an early stage of human hepatocarcinogenesis. Jpn J Cancer Res 88: 1165-1170, 1997.

36. Saito $\mathrm{Y}$, Kanai Y, Sakamoto M, Saito H, Ishii $\mathrm{H}$ and Hirohashi S: Expression of mRNA for DNA methyltransferases and methylCpG-binding proteins and DNA methylation status on $\mathrm{CpG}$ islands and pericentromeric pericentromeric satellite regions during human hepatocarcinogenesis. Hepatology 33: 561-568, 2001.

37. Kanai K, Ushijima S, Kondo Y, Nakanishi Y and Hirohashi S: DNA methyltransferase expression and DNA methylation of $\mathrm{CpG}$ islands and peri-centromeric satellite regions in human colorectal and stomach cancers. Int J Cancer 91: 205-212, 2001.

38. Zuo T, Liu TM, Lan X, Weng YI, Shen R, Gu F, Huang YW, Liyanarachchi S, Deatherage DE, Hsu PY, Taslim C, Ramaswamy B, Shapiro CL, Lin HJL, Cheng ASL, Jin VX and Huang TH: Epigenetic silencing mediated through activated PI3K/AKT signaling in breast cancer. Cancer Res 71: 1752-1762, 2011.
39. Zhang B, Wang $\mathrm{Q}$ and Pan X: MicroRNAs and their regulatory roles in animals and plants. J Cell Physiol 210: 279-289, 2007.

40. Jiang Q, Wang Y, Hao Y, Juan L, Teng M, Zhang X, Li M, Wang $G$ and Liu Y: miR2 Disease: a manually curated database for microRNA deregulation in human disease. Nucleic Acids Res 37: D98-D104, 2009.

41. Meng F, Enson R, Wehbe-Janek H, Ghoshal K, Jacob ST and Patel T: MicroRNA-21 regulates expression of the PTEN tumor suppressor gene in human hepatocellular cancer. Gastroenterology 133: 647-658, 2007.

42. Hatley ME, Patrick DM, Garcia MR, Richardson JA, Duby RB, van Rooij E and Olson EN: Modulation of K-Ras-dependent lung tumorigenesis by MicroRNA-21. Cancer Cell 18: 282-293, 2010 . 